\title{
Cosmic Ray Induced Noise in Gravitational Wave Detectors
}

\author{
R. W. Clay, A. G. K. Smith and J. L. Reid \\ Department of Physics and Mathematical Physics, University of Adelaide, \\ Adelaide, SA 5005, Australia \\ rclay@physics.adelaide.edu.au \\ Received 1996 August 16, accepted 1997 March 21
}

\begin{abstract}
Cosmic radiation is capable of depositing significant quantities of energy and momentum in gravitational wave antennas at times that will generally be random with respect to gravitational signals. Such cosmic ray effects may generate noise signals at detectable levels compared to antenna design thresholds at rates of a few per year but, since antennas will not be capable of absorbing all the cosmic ray energy, it should be readily possible to construct vetoing cosmic ray detectors. For proposed interferometer antennas, a deposition of a few hundred $\mathrm{GeV}$ will produce a detectable noise signal and the veto will require about $100 \mathrm{MeV}$ of energy deposition outside the antenna. We consider here some of the principles involved in the generation of gravitational antenna noise by cosmic ray particles and we describe a veto system to be installed at the Perth gravitational wave observatory.
\end{abstract}

Keywords: cosmic rays - gravitational antennas

\section{Introduction}

Cosmic rays are individual high-energy particles that travel through space from sources both within our Galaxy and in other galaxies. Most of these 'primary' particles are charged nuclei but there is also an important neutral component, particularly the gamma rays. The latter, and perhaps most of the cosmic rays, come from objects which may also produce gravitational signals. We note that it is possible that primary neutrons or gamma rays could carry the same periodicity in their signal.

The spectrum of cosmic ray energies conventionally extends from about $1 \mathrm{GeV}$ up to $100 \mathrm{EeV}$ (1 EeV is $10^{18} \mathrm{eV}$ ). These are practical observational limits and may bear little correspondence to the real energy limits of charged cosmic particles. Between these limits, the energy spectrum is a steep power law with an integral index somewhat below -2 .

The primary cosmic rays are ultra-relativistic with, generally, much more kinetic energy than rest mass energy. As a result, when they interact with the atmosphere of the Earth, many energetic secondary particles are produced which may themselves produce further particles, so that a fraction of the original energy and momentum of the cosmic ray is carried to ground level and may be deposited in the gravitational wave system. The major question is whether or not this deposition of energy and momentum, almost random in time, represents a significant source of noise to the gravitational system, and a further question is whether or not such noise may be economically eliminated. The literature addresses these issues and we discuss here some of the principles involved (see Amaldi \& Pizzella 1986; Ricci 1987; Giazotto 1988; Chiang et al. 1992).

\section{Cosmic Rays at Ground Level}

To a reasonable approximation for most absorbing materials, the interaction properties of cosmic rays can be adequately described by the absorber thickness, measured in grams per square centimetre. Primary cosmic rays interact with the atmosphere (of vertical absorbing depth about $1000 \mathrm{~g} \mathrm{~cm}^{-2}$ ) with a mean free path of below about $100 \mathrm{~g} \mathrm{~cm}^{-2}$. In effect then, all ground-level cosmic rays are secondaries resulting either from the first interaction or from a cascade of secondaries (an extensive air shower, or EAS). The rate of cosmic ray secondary shower cores passing through one square metre of horizontal detector is about one per year at a primary energy of $10^{16} \mathrm{eV}$ and is correspondingly higher (given the steep power-law energy spectrum) at lower energies.

The secondary particles that result from the interactions of primary particles with the atmosphere are conventionally divided into three groups: the hadrons, the muons and the electromagnetic component (electrons, positrons and photons). The origins of these components can be seen by considering the first interaction of a primary cosmic ray with a nucleus in the atmosphere. The mean free path 
for this interaction is below $100 \mathrm{~g} \mathrm{~cm}^{-2}$ (or within the top $10 \%$ of the atmosphere) and the interaction results in the production of secondary hadrons (mainly pions) and the degradation of the energy of the primary particle (typically by $50 \%$ ). Each subsequent interaction of the primary particle adds to the hadron number and degrades the primary energy so that the central region of the EAS develops a core of nuclear-active particles. This core is well collimated and narrow since the hadrons have high longitudinal momentum and have relatively low momentum in the centre of mass.

Pions resulting from nuclear interactions in the core are responsible for the other two shower components. The charged pions may interact or may decay. The decays produce muons (and neutrinos) which are generally assumed to be unlikely to interact further in the atmosphere except by progressive ionisation. It is possible that some high-energy muons may have catastrophic energy loss in a gravitational detector, thus producing a mechanism for inducing noise.

As a result of the high altitude of many of the hadronic processes that generate them and their angle of production, muons spread in a geometrical way through the atmosphere and some can be found over a kilometre from the core, where they dominate the charged-particle density. Also, for the lower-energy primary particles, the central hadronic core may be absorbed before ground level is reached, leaving only remnant muons which then constitute a background of 'unaccompanied' muons. These are numerically the dominant form of sea-level cosmic radiation. Singly, they do not present a serious noise source to current gravity wave detectors. However, it is possible that their arrival time distribution might contain a significant component at the resonant frequency of the antenna.

Neutral pions quickly decay to gamma rays which initiate cascades of electrons and gamma rays fed by bremsstrahlung and pair production until the average energy of the cascade particles drops below the critical energy in air of a few tens of MeV. These cascades consist of many relatively short paths and the cascades reach a form of equilibrium with a characteristic spread from the core (the Moliére radius) of about $80 \mathrm{~m}$ at sea level.

The three shower components then have quite different lateral scales. These are: a few metres for the central hadronic core, about $100 \mathrm{~m}$ for the electromagnetic component and several hundred metres for the muons. The electromagnetic component dominates numerically when integrated over the whole shower but the highest-energy particles are found near the core, which has the greatest particle density per square metre. This central core, or individual particles within it, presents the greatest potential for inducing noise in gravitational systems.

\section{Effect of Cosmic Ray Showers on Gravitational Detectors}

There would appear to be two likely modes by which cosmic rays may induce noise signals in gravitational wave antennas. These are associated with the ways in which energy and momentum deposition can respectively affect the antenna. Energy deposition in the antenna may cause heating and a thermal expansion of the antenna components. Amaldi \& Pizzella (1986) note that resonant bar antennas might aim for a sensitivity of the order of $10^{-7} \mathrm{~K}$ and that rather simple arguments equate this temperature to a typical energy deposition of a few $\mathrm{GeV}$ due to cosmic radiation. Also, in an interferometer, the momentum of the incoming particle may be transferred to a mirror and cause it to move on its pendulum support. The relative importance of these two effects is detector-dependent and either could be important. Remarkably, one way or another, a few hundred $\mathrm{GeV}$ of energy deposition seems to be capable of producing significant noise in presently proposed antennas, and a few $\mathrm{GeV}$ may be important for antennas of the following generation.

Giazotto (1988) has considered the effect of momentum deposition on internal oscillations of the mirror and its surface in an interferometer, and concluded that such an effect is dominated by the heating term [see his equation (7)]. However, the effect of momentum deposition on pendulum oscillation of the mirror needs to be considered, and an energy of the order of a thousand $\mathrm{GeV}(1 \mathrm{TeV})$ would seem to be a threshold for such a noise effect to be important.

We can check the likely rate of potential cosmic ray noise events from the measured cosmic ray density spectrum ( e.g. Ashton \& Parvaresh 1975). This describes the rate at which various densities of particles (in particles per square metre) are detected. The density spectrum is closely related to the cosmic ray energy spectrum and has a similar power-law shape. If we assume that near the core each particle carries about $100 \mathrm{MeV}$ (e.g. Dawson 1995), we are interested in the frequency of bursts containing 10000 particles or more in order to have $1 \mathrm{TeV}\left(10^{12} \mathrm{eV}\right)$ passing into the detector. This is expected roughly once in 5000 hours or a few times per year.

\subsection{Thermal Effects}

When a cosmic ray event deposits energy in a bar, the energy is degraded to heat and causes the bar to expand. Grassi Strini, Strini \& Tagliaferri (1980) derived the amplitude of the fundamental mode of the bar under such heating and this is close to the magnitude of thermal expansion of the bar but, naturally, depends on the way that the excitation occurs, particularly the position of the heating site. 
For a system such as a resonant bar, the effect can be large compared with the sensitivity required to detect a distant source.

\subsection{Momentum Effects}

Giazotto (1988) has examined in some detail the effect on an interferometer of the deposition of momentum by cosmic rays. We can examine such a process in a simple way by considering the effect of an impulsive deposition of momentum $(E / c)$, where $E$ is the cosmic ray energy deposited. (There will be a geometrical factor to determine the horizontal component of this momentum for a horizontal interferometer.) The resulting change in speed of the mirror (mass $M$ ) will be $(E / c) / M$ and the displacement in time $\mathrm{d} t$ will be this speed times $\mathrm{d} t$. The displacement expressed as a fraction of the interferometer arm can then be expressed as a measurability limit, $h$. As Giazotto found, using typical interferometer parameters, this limit for $h$ is about $10^{-25} E$, where $E$ is the energy deposition in $\mathrm{GeV}$ and $\mathrm{d} t$ is taken to be of the order of the pendulum oscillation time. Thus a deposition of about $1 \mathrm{TeV}$ would again give a threshold signal.

\section{Interaction of Cosmic Ray Showers with Material}

Most of the energy and momentum of the primary cosmic ray particle ends in some form of ionisation, or the photoelectric effect for the photon component. The rate of energy loss is most significant for the relatively. low-energy electromagnetic component and least significant for the muons which, unless they have very high energies, lose energy only by ionisation.

The primary cosmic ray particle has a mean free path of up to $100 \mathrm{~g} \mathrm{~cm}^{-2}$ and loses roughly half of its energy at each interaction. As a result, to a first approximation, the primary energy is degraded by 1000 times in reaching sea level. Individual charged shower particles will typically lose energy by ionisation at a rate of about $2 \mathrm{MeV}\left(\mathrm{g} \mathrm{cm}^{-2}\right)^{-1}$ but may have other energy loss processes.

In considering noise processes for gravitational wave antennas, current sensitivity limits require that we examine processes that can deposit energy (or momentum $\times c$ ) at the level of hundreds of $\mathrm{GeV}$ at rates of at least one per year. This means that we may have to consider the possibility of occasional rare energy deposition mechanisms. The likely processes seem to be associated with hadronic cores or extremely energetic muons.

\subsection{Hadronic Cores}

The hadronic core of the shower carries energy through energetic nuclear-active particles. As such, the core is readily detectable using conventional and inexpensive particle detectors, and veto techniques for a small event flux in the energy range of interest should be straightforward. If we consider the core energy to be degraded by about 1000 times in traversing the atmosphere, we can see that, if we require at least $100 \mathrm{GeV}$ of deposited energy, we need to be considering primary particles with energies above $100 \mathrm{TeV}$. Such primaries arrive with a frequency of about ten per day over the roughly one square metre of resonant bar area. At a more extreme level, we would expect a core energy of about $10 \mathrm{TeV}$ once per year. Roughly half of the $10 \mathrm{TeV}$ will be in the remaining central particle and one might expect to see a core with 50,000 particles about once per year. This is indeed what is found.

Core hadrons are energetic and are thus rather penetrating. They will lose a substantial fraction, but not all, of their energy in traversing a few tens of $\mathrm{g} \mathrm{cm}^{-2}$ of gravitational detector. From the measured spectrum of charged hadrons (Allkofer \& Grieder 1984) we conclude that a once-a-year hadron impulse on a gravitational detector will deposit about $1 \mathrm{TeV}$ of energy and the rate of impulses of smaller magnitude will increase roughly as an inverse power law with an index of about $2 \cdot 0$. Such impulses may well be detectable as noise in the antenna. Particles in the shower core are penetrating, and substantial particle energy will be readily detectable above or below (or both) the antenna using conventional particle detectors.

\subsection{High Energy Muons}

EAS have muons as one of their significant components and the majority of the overall cosmic ray background consists of unaccompanied muons. These arrive at sea level at a rate of about one per square $\mathrm{cm}$ per minute (or some tens of particles per second through a typical modestly sized component part of a gravitational detection system). Clearly, rates such as this cannot be vetoed for systems that have bandwidths only up to the $\mathrm{kHz}$ range.

Air-shower muons have characteristic energies in the $\mathrm{GeV}$ range and so typical individual muons will not be a major gravitational noise problem. However, it is possible that Fourier components may exist in the random arrival distribution that correspond to the resonant frequency of the gravitational system, or a very rare energetic muon may deposit a large fraction of its energy in the antenna. The former problem has been discussed by Giazotto (1988) and appears to be unimportant. Based on Monte Carlo simulations, rather more discussion of the latter problem has appeared in the literature and it is worth identifying the basis for this noise mechanism.

Muons result from the decay of pions that are produced when core shower particles (most likely the degraded incident particle) interact with atmospheric nuclei. The resulting muon energy spectrum will then be related to the primary energy spectrum in its structure but will be somewhat steeper due to a deficiency in muons at the highest energies. 
This is because the number of pions produced in an interaction rises somewhat with energy and hence the relative energy per pion (and the resulting muon) drops. Also, due to time dilation, the pions have a longer lifetime at higher energies and are less likely to decay and produce muons before they interact. Again, the result is a reduction in the possible number of high-energy muons.

We now need to see how often a muon of sufficient energy to produce a significant noise pulse will pass through the detector, and then determine how often such a muon will interact close to the detector and actually deposit energy. If we remember that perhaps half of the incident energy is retained by the primary particle and the rest is distributed between the pions (albeit non-uniformly), we might identify perhaps $20 \%$ of the incident energy going to the most energetic (and most important, from our point of view) muon. Also, at a fixed primary energy, the cosmic ray nuclei have a mixed composition and, if we are concentrating on high-energy muons, we are only interested in the proton primary component. This is because other, more complex, nuclei will produce more, but lower-energy, muons. If we take shower primaries with an energy of $1 \mathrm{TeV}$, we can say that the flux of muons produced with energies above $0.2 \mathrm{TeV}$ is about $10^{7}$ per square metre per year. As we noted earlier, an energy deposition of this order is capable of producing significant noise in presently proposed antennas.

Muons generally lose energy by ionisation. However, at energies above a few hundred $\mathrm{GeV}$ (i.e. our selected energy and above), pair production, bremsstrahlung and photonuclear interactions result in large energy loss events. We need to consider the probability of such an event occurring in the gravitational detector for a muon of this energy, noting that already, by selecting these energetic muons, we are examining only one muon in 30,000 .

The scale factor for energy deposition with these large energy processes is about $2.5 \times 10^{5} \mathrm{~g} \mathrm{~cm}^{-2}$ in rock, which we will take as a worst case (the figure for hydrogen is perhaps ten times greater). If the gravitational detector has an absorption thickness of $10-100 \mathrm{~g} \mathrm{~cm}^{-2}$ we would thus estimate that there is a probability of about one in $10^{4}$ of our muon interacting in this way in the detector. We are now looking at about 1000 potential events per year per square metre.

The question now is how much energy will actually be deposited in the gravitational detector. The detector will absorb some of the energy but, with a limited thickness and typical ionisation loss rates of charged secondaries of $2 \mathrm{MeV}$ per $\mathrm{g} \mathrm{cm}^{-2}$ or a few hundred $\mathrm{MeV}$ per particle per detector thickness, a significant fraction will leak out. The leaking energy could easily be used for a veto through the use of a large-area detector placed below the antenna structure. The signature of such a muon-initiated event would be a large detector signal resulting from the cascading of secondary particles in the antenna and its housing. It need not be accompanied by a signal in other detectors. If we assume $10 \%$ absorption, then to get an energy deposition of $0.2 \mathrm{TeV}$, we are looking at a rate of about 1 to 10 events per detector per year.

We thus conclude that, for presently proposed antennas, shower cores or the effects of secondary particles from the interaction of single high-energy muons will provide noise pulses at a rate of about a few per year but these pulses will almost certainly be accompanied by detectable signals in a local veto particle detector. These noise pulses should be detectable and could be used as a test of the true antenna sensitivity after a detailed calculation with the known antenna characteristics.

\section{Cosmic Ray Veto Methods}

Perhaps the most powerful noise elimination method is to find a coincidence between two antennas which are themselves triggering at a slow rate. Since there will be accidental coincidences, it is still important to address all possible techniques for reducing the noise contamination of individual data sets. To the extent that local cosmic radiation causes noise (remembering that it also may come as gamma rays from the source to be detected and may thus retain characteristics of the source), a charged-particle detector monitoring cosmic ray bursts will be a useful noise veto. Additionally, Chiang, Michelson \& Price (1992) have noted that the lowest allowed moment of gravitational radiation is the quadrupole and the second longitudinal mode cannot be excited. Their simulation indicates that a significant reduction in noise from cosmic ray muons may be achieved if a second longitudinal mode veto is also applied.

We have seen that energy deposition of a few hundred $\mathrm{GeV}$ in a gravitational antenna by a cosmic ray can be a significant source of noise at a rate of a few events per year. We have also seen that this deposition can occur through the interaction of an air-shower core with the detector or through the rare interaction of a very energetic muon. In the latter case, energy is deposited through the production and absorption of secondary particles and in the former case by the interactions and absorption of existing particles. In both cases, an antenna component of $10-100 \mathrm{~g} \mathrm{~cm}^{-2}$ thickness will not absorb all the incident energy and some will flow through. This remaining energy, or the incident energy for a core, can be detected and the detection can flag the experimenter to ignore any coincident signal from the gravitational antenna. Large-area (a few square metres) cosmic ray detectors are simple and cheap (approximately $\$ 10,000$ ) to build and 
can readily respond to signals below $10 \mathrm{MeV}$. Such a detector responding to an energy deposition of, say, $100 \mathrm{MeV}$ below an antenna could provide an efficient and cost-effective conservative veto. This level of $100 \mathrm{MeV}$ would result in a veto trigger typically at a rate of one event every few hours with a detector area of about one square metre.

Coccia et al. (1995) have described a cosmic ray veto system for the NAUTILUS gravitational wave detector. This detector uses a $2300 \mathrm{~kg}$ aluminium bar, and a few events per day are expected to result from cosmic ray effects. Their cosmic ray detector system consists of layers of limited streamer tubes and the trigger logic responds to high-energy muons and hadrons as well as extensive air showers. Coincidences are taken between layers of tubes above and below the gravitational wave detector. ADC saturation sets an upper limit of about 1000 particles for any given tube.

We note that if a future generation of antennas approaches the quantum limit, then a cosmic ray system will be unable to assume that substantial energy can leak through the detector housing. Any veto system will need to be installed within the detector itself.

\section{Proposed Veto Array for the Perth Antenna}

We have provided a two-scintillation-detector coincidence system for use with the University of Western Australia niobium-bar gravitational wave antenna to veto possible events in coincidence with cosmic ray showers. This has been available for some time. It has a primary particle threshold energy for shower detection of about $10 \mathrm{TeV}$. We have now designed an upgraded system based on our Thebarton Array (Smith \& Clay 1996) which will provide an air shower arrival trigger and will also provide an estimate of the number of particles crossing the antenna bar for each event. This requirement is rather different to conventional air shower measurements, which concentrate on estimating the total particle content of the shower. Here, we wish to have an estimate of the particle density in the antenna. This is similar to measuring the particle density spectrum (also known as the burst spectrum) over the whole of the antenna.

The design is based on five scintillation detectors, each $400 \mathrm{~mm}$ square. We propose to have the detectors at the corners and the centre of a square with $5 \mathrm{~m}$ sides. Since we are interested in determining the density of particles up to quite high levels, we will set the individual detector thresholds at about six particles, which will give an array primary energy threshold at about $500 \mathrm{TeV}$ for threefold coincidences. We anticipate an event rate of about one per hour. It will also be possible to trigger the system on a single large particle density in the detector below the gravitational antenna. If that detector has a threshold set at 15 particles, we would again expect a trigger rate of about one per hour with a primary energy threshold of about $200 \mathrm{TeV}$ (Ashton \& Parvaresh 1975).

Most array triggers will include the central detector, which will be placed below the antenna bar. As a result there will be a direct estimate of the particle number in the antenna with a range up to 10000 particles. If that detector were to saturate, the remaining detectors would provide density information that could be used to estimate the density in an independent way to better than a factor of two. Those detectors would also serve as a check on the central density, which will be rather dependent on the exact impact point of the air shower core.

\section{Conclusions}

Cosmic ray effects present possible sources of noise for gravitational wave antennas at event rates that are significant for the successful operation of the detectors (a few per year). It is possible to construct veto systems that will respond to large particle densities in the vicinity of an antenna, either from muons, hadrons or extensive air showers. We have designed such a system, based on our previous Thebarton air shower array, which we expect to install shortly at the Perth gravitational wave observatory.

\section{Acknowledgments}

David Blair is thanked for introducing us to this problem and Peter Veitch for introducing us to background literature in the area and for very enlightening discussions.

Allkofer, O. C., \& Grieder, P. K. F. 1984, Cosmic Rays on Earth (Fachinformationszentrum Energie, Physik, Mathematik $\mathrm{GmbH}$ )

Amaldi, E., \& Pizzella, G. 1986, Nuovo Cimento, 9C, 612 Ashton, F., \& Parvaresh, A. 1975, Proc. 14th Int. Cosmic Ray Conf. (Munich: Max Planck Inst. fur Extraterrestriche Physik), 8, 2719

Chiang, J., Michelson, P., \& Price, J. 1992, Nucl. Instrum. Meth., A311, 603

Coccia, E., et al. 1995, Nucl. Instrum. Meth., A355, 624

Dawson, B. R. 1995, Adelaide Design Workshop on Techniques for the Study of Cosmic Rays with Energies above $10^{19}$ eV, ed. R. W. Clay \& B. R. Dawson (Adelaide University), 47

Giazotto, A. 1988, Phys. Lett. A128, 241

Grassi Strini, A. M., Strini, G., \& Tagliaferri, G. 1980, J. Appl. Phys., 51, 948

Ricci, F. 1987, Nucl. Instrum. Meth., A260, 491

Smith, A. G. K., \& Clay, R. W. 1997, Aust. J. Phys., 50, 827 